\title{
Spectrum of the Schrödinger operator in a perturbed periodically twisted tube
}

\author{
P. Exner ${ }^{a, b}$ and H. Kovařík ${ }^{a, c}$ \\ a) Department of Theoretical Physics, Nuclear Physics Institute, Czech Academy \\ of Sciences, CZ-25068 Řez near Prague \\ b) Doppler Institute, Czech Technical University, Břehová 7, CZ-11519 Prague \\ c) Institute for Analysis, Dynamics and Modeling, Faculty of Mathematics and \\ Physics, Stuttgart University, PF 8011 40, D-70569 Stuttgart. Germany \\ exner@ujf.cas.cz, kovarik@mathematik.uni-stuttgart.de
}

\begin{abstract}
We study Dirichlet Laplacian in a screw-shaped region, i.e. a straight twisted tube of a non-circular cross section. It is shown that a local perturbation which consists of "slowing down" the twisting in the mean gives rise to a non-empty discrete spectrum.
\end{abstract}

\section{Introduction}

Existence of geometrically-induced bound states in infinitely extended regions of tubular shape was noticed at the end of the eighties, first in the twodimensional situation [7, and studied intensively since then - see [3, 9, 11, 13, and more recently [2, 6]. The effective interaction which lead in these cases to the existence of a discrete spectrum came from tube bends. If the tube is embedded in $\mathbb{R}^{d}$ with $d \geq 3$ one can ask about the effect of its twisting on the spectrum. In the work quoted above the tubes considered were either circular or twisted in a particular way aligned with the tube axis torsion; in that case the twist did not affect the result in the leading order.

Investigations going beyond this special case were done only recently. Inspired by the existence of a magnetic Hardy-type inequality in waveguides [4] the authors of [5] analyzed the generic case of a local tube twist and found 
that it gives rise to a repulsive effective interaction which can destroy weakly bound states coming from other perturbations. In this letter we push this idea further and study a twist which extends along the whole tube.

As a repulsive interaction it rises the essential spectrum threshold. At the same time, if we perturb such a screw-shaped tube locally in such a way that the repulsion is weakened, e.g. by a local slowdown of the twist, one may expect a binding effect. We show, under mild regularity assumptions, that it is indeed the case and that it is sufficient if the twisting is slowed down locally in the mean. Moreover, a natural analogy with one-dimensional Schrödinger operator theory suggests that the effect might survive in the critical case when the mean value of the twist variation is zero. It is not a reliable guide, of course, because sometimes in similar situations critical bound states are absent [1], nevertheless, here we are able to demonstrate that discrete spectrum for a critical twist perturbation is still non-empty.

Let us review the contents of the letter. We will introduce the needed notation in the next section, then we will analyze the spectrum in the periodic case. Our main results are given in Section 4, specifically in Theorems 2 and 3 for the non-critical and critical situation, respectively.

\section{Preliminaries}

First we fix the notation. Let $\omega$ be an open bounded and connected set in $\mathbb{R}^{2}$ and let $\theta$ be a differentiable function from $\mathbb{R}$ to $\mathbb{R}$. For $s \in \mathbb{R}$ and $t:=\left(t_{2}, t_{3}\right) \in \omega$ we define the mapping $\mathcal{L}$ from $\mathbb{R} \times \omega$ to $\mathbb{R}^{3}$ by

$$
\mathcal{L}(s, t)=\left(s, t_{2} \cos \theta(s)+t_{3} \sin \theta(s), t_{3} \cos \theta(s)-t_{2} \sin \theta(s)\right) .
$$

The image $\mathcal{L}(\mathbb{R} \times \omega)$ is a tube in $\mathbb{R}^{3}$ which is twisted unless the function $\theta$ is

constant. A case of particular interest is a screw-shaped tube corresponding to a linear $\theta$. We fix a positive constant $\beta_{0}$ and define the tube $\Omega_{0}$ by

$$
\Omega_{0}:=\mathcal{L}_{0}(\mathbb{R} \times \omega)
$$

where

$$
\mathcal{L}_{0}(s, t):=\left(s, t_{2} \cos \left(\beta_{0} s\right)+t_{3} \sin \left(\beta_{0} s\right), t_{3} \cos \left(\beta_{0} s\right)-t_{2} \sin \left(\beta_{0} s\right)\right) ;
$$

it will play role of the unperturbed system. The operator we will be concerned with is the Dirichlet Laplacian $H_{0}$ on $L^{2}\left(\Omega_{0}\right)$, i.e. the self-adjoint operator 
associated with the closed quadratic form

$$
Q_{0}[\psi]:=\int_{\Omega_{0}}|\nabla \psi|^{2} \mathrm{~d} s \mathrm{~d} t, \quad \forall \psi \in D\left(Q_{0}\right)=\mathcal{H}_{0}^{1}\left(\Omega_{0}\right)
$$

\section{Spectrum of $H_{0}$}

Given $\psi \in C_{0}^{\infty}(\mathbb{R} \times \omega)$ it is useful to introduce the following shorthand,

$$
\psi_{\tau}^{\prime}:=t_{2} \partial_{t_{3}} \psi-t_{3} \partial_{t_{2}} \psi
$$

A simple substitution of variables shows that

$$
Q_{0}[\psi]=\int_{\mathbb{R} \times \omega}\left|\nabla_{t} \psi\right|^{2}+\left|\partial_{s} \psi+\beta_{0} \psi_{\tau}^{\prime}\right|^{2} \mathrm{~d} s \mathrm{~d} t
$$

where

$$
\nabla_{t} \psi:=\left(\partial_{t_{2}} \psi, \partial_{t_{3}} \psi\right)
$$

In other words, the operator $H_{0}$ acts on its domain in $L^{2}\left(\Omega_{0}\right)$ as

$$
H_{0}=-\partial_{t_{2}}^{2}-\partial_{t_{3}}^{2}+\left(-i \partial_{s}-i \beta_{0}\left(t_{2} \partial_{t_{3}}-t_{3} \partial_{t_{2}}\right)\right)^{2}
$$

Since $\beta_{0}$ is independent of $s$ we are able to employ a partial Fourier transformation $\mathcal{F}_{s}$ given by

$$
\left(\mathcal{F}_{s} \psi\right)(p, t)=\hat{\psi}(p, t)=\frac{1}{\sqrt{2 \pi}} \int_{\mathbb{R}} e^{-i p s} \psi(s, t) \mathrm{d} s,
$$

which allows us ro rewrite the quadratic form as

$$
Q_{0}[\hat{\psi}]=\int_{\mathbb{R} \times \omega}\left|\nabla_{t} \hat{\psi}\right|^{2}+\left|i p \hat{\psi}+\beta_{0} \hat{\psi}_{\tau}^{\prime}\right|^{2} \mathrm{~d} p \mathrm{~d} t
$$

for a suitably regular $\psi$. Since the transformation $\mathcal{F}_{s}$ extends to a unitary operator on $L^{2}(\mathbb{R} \times \omega)$, the operator $H_{0}$ is unitarily equivalent to the direct integral

$$
\int_{\mathbb{R}}^{\oplus} h(p) \mathrm{d} p
$$

with the fibre operator

$$
h(p)=-\partial_{t_{2}}^{2}-\partial_{t_{3}}^{2}+\left(p-i \beta_{0}\left(t_{2} \partial_{t_{3}}-t_{3} \partial_{t_{2}}\right)\right)^{2}
$$


on $L^{2}(\omega)$ subject to Dirichlet boundary conditions at $\partial \omega$. Introducing the polar coordinates $(r, \alpha)$ on $\omega$, we can rewrite $h(p)$ as follows

$$
h(p)=-\Delta_{D}^{\omega}+\left(p-i \beta_{0} \partial_{\alpha}\right)^{2},
$$

where $-\Delta_{D}^{\omega}$ denotes the Dirichlet Laplacian in $r$ and $\alpha$. Since $h(p)$ is a sum of $-\Delta_{D}^{\omega}$ and a positive perturbation, it follows easily from the minimax principle that its spectrum is purely discrete. Let us denote the eigenvalues of $h(p)$ by $\varepsilon_{n}(p)$ and the respective eigenfunctions by $\psi_{n}(p)$, i.e.

$$
h(p) \psi_{n}(p)=\varepsilon_{n}(p) \psi_{n}(p) .
$$

Lemma 1. Every $\varepsilon_{n}(\cdot), n \in \mathbb{N}$, is a real-analytic function of $p$ and

$$
\lim _{p \rightarrow \pm \infty} \varepsilon_{n}(p) \rightarrow \infty
$$

Proof. It is not difficult to check that the quadratic form associated with the operator $h(0)$ defined on the form domain $\mathcal{H}_{0}^{1}\left(\Omega_{0}\right)$ is non-negative and closed. This implies that $h(0)$ is self-adjoint on its natural domain which we denote as $D(0)$. Let us formally expand the square in (6) and write $h(p)$ as

$$
h(p)=h(0)+p^{2}-2 i p \beta_{0} \partial_{\alpha} .
$$

Denote the resolvent of $h(0)$ at a point $z \in \mathbb{C}$ by $R_{z}$, i.e. $R_{z}=(h(0)-z)^{-1}$. Then we have for any $\varphi \in C_{0}^{\infty}(\omega)$ the following estimate

$$
\begin{aligned}
\left\|\partial_{\alpha} \varphi\right\|^{2} & \leq(\varphi, h(0) \varphi)=\left(R_{z}(h(0)-z) \varphi, h(0) \varphi\right) \\
& \leq\left\|R_{z}\right\|\|h(0) \varphi\|^{2}+|z|\left(\varphi, R_{\bar{z}} h(0) \varphi\right) \\
& \leq C(z)\|h(0) \varphi\|^{2}+|z|^{2} C(z)\|\varphi\|^{2}
\end{aligned}
$$

where $C(z) \rightarrow 0$ as $\Im z \rightarrow \infty$. Consequently, $i \partial_{\alpha}$ is $h(0)$-bounded with the relative bound zero which implies that the domain of $h(p)$ coincides with $D(0)$ and the vector $h(p) \phi$ is analytic as a function of $p$ for every $\phi \in D(0)$ (since $p^{2}$ is clearly analytic). From [10, pp. 375 and 385, it thus follows that $\{h(p): p \in \mathbb{R}\}$ is a self-adjoint analytic family of type $A$ and that all the $\varepsilon_{n}(\cdot)$ are real-analytic functions of $p$.

To prove the second statement of the lemma, let us first define the crosssection radius with respect to the rotation axis,

$$
a:=\sup _{t \in \omega}|t|
$$


We observe that for any $\varphi \in C_{0}^{\infty}(\omega)$ we have a trivial pointwise inequality,

$$
\left|2 p \beta_{0} \bar{\varphi} \partial_{\alpha} \varphi\right| \leq p^{2} \frac{\beta_{0}^{2}}{\beta_{0}^{2}+a^{-2}}|\varphi|^{2}+\left(\beta_{0}^{2}+a^{-2}\right)\left|\partial_{\alpha} \varphi\right|^{2},
$$

which implies that

$$
\begin{aligned}
& (\varphi, h(p) \varphi)=\int_{\omega}\left(\left|\partial_{r} \varphi\right|^{2}+\frac{1}{r^{2}}\left|\partial_{\alpha} \varphi\right|^{2}+\left|\left(p-i \beta_{0} \partial_{\alpha}\right) \varphi\right|^{2}\right) r \mathrm{~d} r \mathrm{~d} \alpha \\
& \quad \geq \int_{\omega}\left(\left|\partial_{r} \varphi\right|^{2}+a^{-2}\left|\partial_{\alpha} \varphi\right|^{2}+p^{2}|\varphi|^{2}-\left|2 p \beta_{0} \bar{\varphi} \partial_{\alpha} \varphi\right|+\beta_{0}^{2}\left|\partial_{\alpha} \varphi\right|^{2}\right) r \mathrm{~d} r \mathrm{~d} \alpha \\
& \quad \geq \frac{1}{1+a^{2} \beta_{0}^{2}} p^{2} \int_{\omega}|\varphi|^{2} r \mathrm{~d} r \mathrm{~d} \alpha
\end{aligned}
$$

this in turn yields the sought result.

It is clear from (5) that the spectral threshold of $h(0)$ cannot be lower than that of $-\Delta_{D}^{\omega}$. It has been shown in [5] that the inequality is sharp,

$$
E:=\inf \sigma(h(0))>\inf \sigma\left(-\Delta_{D}^{\omega}\right)
$$

whenever $\omega$ is not rotationally symmetric. This follows, by the way, also from our Lemma $2 b$ which will be proved below.

Our aim is to show that this quantity determines the spectral threshold of our original Hamiltonian, in other words, $E=\inf \sigma\left(H_{0}\right)$. To this end let us denote by $f$ the real-valued eigenfunction of $h(0)$ associated with the eigenvalue $E=\varepsilon_{1}(0)$, i.e.

$$
h(0) f=-\Delta_{D}^{\omega} f-\beta_{0}^{2} \partial_{\alpha}^{2} f=E f .
$$

Then we can make the following claim.

Lemma 2. Let $f$ be given by (9). Then

(a) $f$ is strictly positive in $\omega$.

(b) $\int_{\omega}\left|f_{\tau}^{\prime}\right|^{2} \mathrm{~d} t=\int_{\omega}\left|\partial_{\alpha} f\right|^{2} \mathrm{~d} t>0$ provided $\omega$ is not rotationally symmetric.

Proof. To prove the positivity of $f$ it is enough to show that the semigroup $e^{-t h(0)}$ is positivity improving for all $t>0$, see [12, Thm XIII.44], that is, we 
have to show that $e^{-t h(0)}$ maps every positive function in $\omega$ into a strictly positive function in $\omega$. Since $-\Delta_{D}^{\omega}$ commutes with $\partial_{\alpha}^{2}$, we get

$$
e^{-t h(0)}=e^{t \Delta_{D}^{\omega}} e^{t \beta_{0}^{2} \partial_{\alpha}^{2}}
$$

However, it follows easily from [12, Thm XIII.50] that $e^{t \beta_{0}^{2} \partial_{\alpha}^{2}}$ is positivity preserving for all $t>0$, i.e. it maps every positive function into a positive function. Now note that since $-\Delta_{D}^{\omega}$ has a strictly positive ground state, $e^{t \Delta_{D}^{\omega}}$ is positivity improving for all $t>0$ by [12, Thm XIII.44]. Hence given a positive function $g$ in $\omega$, we know that $e^{t \beta_{0}^{2} \partial_{\alpha}^{2}} g$ is positive, which means that $e^{-t h(0)} g$ is strictly positive; this proves the first statement of the Lemma.

The second statement is an immediate consequence of the first one. Let $B$ be the biggest circle (i.e., the one with the biggest radius) centred at the origin, such that $B \subset \bar{\omega}$. Denote its complement in $\bar{\omega}$ by $B^{c}$. By assumption we know that $B^{c} \neq \emptyset$. Since $f$ satisfies Dirichlet boundary conditions on $\partial \omega$ and is strictly positive inside $\omega$, it follows that $\left|\partial_{\alpha} f\right|$ is strictly positive in almost every point of $B^{c} \cap \partial \omega$, where $\partial \omega$ is not a part of a circle centred at the origin. This "non-circular" part is, of course, a positive measure set, hence using the differentiability of $f$ we can find a neighbourhood of $B^{c} \cap \partial \omega$ with a positive Lebesgue measure on which $\left|\partial_{\alpha} f\right|>0$.

Remark: The first statement of Lemma 2 also follows from [8, Thm. 8.38].

Now we are able to determine the spectrum of the free operator.

Theorem 1. The spectrum of $H_{0}$ is purely absolutely continuous and covers the half-line $[E, \infty)$, where $E$ is the lowest eigenvalue of $h(0)$.

Proof. From (4) and Lemma 1]we know that the spectrum of $H_{0}$ is absolutely continuous and that $[E, \infty) \subset \sigma\left(H_{0}\right)$. It remains to show that

$$
(-\infty, E) \cap \sigma\left(H_{0}\right)=\emptyset \text {. }
$$

Using the fact that the ground-state eigenfunction $f$ is strictly positive in $\omega$, we can decompose any $\psi \in C_{0}^{\infty}(\omega)$ as

$$
\psi(s, t)=f(t) \varphi(s, t) .
$$

We use the fact $f$ is real-valued and integrate by parts to get

$$
\begin{aligned}
Q_{0}[\psi]-E\|\psi\|^{2}= & \int_{\mathbb{R} \times \omega}\left(f^{2}\left|\nabla_{t} \varphi\right|^{2}-\left(\Delta_{D}^{\omega} f\right) f|\varphi|^{2}+f^{2}\left|\partial_{s} \varphi\right|^{2}\right. \\
& +\beta_{0} f \partial_{\alpha} f\left(\partial_{s} \bar{\varphi} \varphi+\bar{\varphi} \partial_{s} \varphi\right)+\beta_{0} f^{2}\left(\partial_{s} \bar{\varphi} \partial_{\alpha} \varphi+\partial_{\alpha} \bar{\varphi} \partial_{s} \varphi\right) \\
& \left.+\beta_{0}^{2} f^{2}\left|\partial_{\alpha} \varphi\right|^{2}-\beta_{0}^{2}\left(\partial_{\alpha}^{2} f\right) f|\varphi|^{2}-E f^{2}|\varphi|^{2}\right) \mathrm{d} s \mathrm{~d} t .
\end{aligned}
$$


Since

$$
\int_{\mathbb{R}}\left(\partial_{s} \bar{\varphi} \varphi+\bar{\varphi} \partial_{s} \varphi\right) \mathrm{d} s=0
$$

and

$$
-\Delta_{D}^{\omega} f-\beta_{0}^{2} \partial_{\alpha}^{2} f-E f=0,
$$

see (9), we finally obtain

$$
Q_{0}[\psi]-E\|\psi\|^{2}=\int_{\mathbb{R} \times \omega} f^{2}\left(\left|\nabla_{t} \varphi\right|^{2}+\left|\partial_{s} \varphi+\beta_{0} \varphi_{\tau}^{\prime}\right|^{2}\right) \mathrm{d} s \mathrm{~d} t \geq 0
$$

This implies (10).

\section{Local perturbations of the twisting}

After analyzing the "free" case, where the twisting velocity $\dot{\theta}$ was constant, we want to look now what will happen if the translation invariance of our tube is broken. We will suppose that the velocity of the twisting is given by

$$
\dot{\theta}(s)=\beta_{0}-\beta(s),
$$

where $\beta(\cdot)$ is a bounded function such that $\operatorname{supp} \beta \subset\left[-s_{0}, s_{0}\right]$ for some $s_{0}>0$. Let $\Omega_{\beta}$ denote the corresponding tube being defined by

$$
\Omega_{\beta}:=\mathcal{L}(\mathbb{R} \times \omega),
$$

where $\mathcal{L}$ refers to the twisting obtained by integration of (12). We use the symbol $H_{\beta}$ for the Dirichlet Laplacian on $L^{2}\left(\Omega_{\beta}\right)$ and

$$
Q_{\beta}[\psi]:=\int_{\Omega_{\beta}}|\nabla \psi|^{2},
$$

will be the associated quadratic form with the form domain $D\left(Q_{\beta}\right)=\mathcal{H}_{0}^{1}\left(\Omega_{\beta}\right)$. Since the support of the perturbation $\beta(s)$ is compact, it is straightforward to check that

$$
\sigma_{\text {ess }}\left(H_{\beta}\right)=\sigma_{\text {ess }}\left(H_{0}\right)=[E, \infty) .
$$

Our main result says that if the tube twisting is locally slowed down in the mean, the discrete spectrum of $H_{\beta}$ is non-empty. 
Theorem 2. Assume that $\omega$ is not rotationally symmetric and that

$$
\int_{-s_{0}}^{s_{0}}\left(\dot{\theta}^{2}(s)-\beta_{0}^{2}\right) \mathrm{d} s<0,
$$

where $\dot{\theta}(\cdot)$ is given by (12). Then the operator $H_{\beta}$ has at least one eigenvalue of finite multiplicity below the threshold of the essential spectrum.

Proof. Following the idea of [9] we start constructing a trial function from a transverse eigenfunction corresponding to the bottom of the essential spectrum. Given $\delta>0$ we put $\Psi_{\delta}(s, t)=f(t) \varphi(s)$, where

$$
\varphi(s)= \begin{cases}e^{\delta\left(s_{0}+s\right)} & \text { if } \quad s \leq-s_{0} \\ 1 & \text { if } \quad-s_{0} \leq s \leq s_{0} \\ e^{-\delta\left(s-s_{0}\right)} & \text { if } \quad s \geq s_{0}\end{cases}
$$

It is easy to see that $\Psi_{\delta} \in D\left(Q_{\beta}\right)$. A straightforward calculation then gives

$$
Q_{\beta}\left[\Psi_{\delta}\right]-E\left\|\Psi_{\delta}\right\|^{2}=\delta\|f\|_{L^{2}(\omega)}^{2}-\left\|f_{\tau}^{\prime}\right\|_{L^{2}(\omega)}^{2} \int_{-s_{0}}^{s_{0}}\left(\dot{\theta}^{2}(s)-\beta_{0}^{2}\right) \mathrm{d} s
$$

and

$$
\left\|\Psi_{\delta}\right\|^{2}=\left(\delta^{-1}+2 s_{0}\right)\|f\|_{L^{2}(\omega)}^{2} .
$$

For $\delta \rightarrow 0$ we then get

$$
\frac{Q_{\beta}\left[\Psi_{\delta}\right]-E\left\|\Psi_{\delta}\right\|^{2}}{\left\|\Psi_{\delta}\right\|^{2}}=\delta \frac{\left\|f_{\tau}^{\prime}\right\|_{L^{2}(\omega)}^{2}}{\|f\|_{L^{2}(\omega)}^{2}} \int_{-s_{0}}^{s_{0}}\left(\dot{\theta}^{2}(s)-\beta_{0}^{2}\right) \mathrm{d} s+\mathcal{O}\left(\delta^{2}\right) .
$$

Thus in view of Lemma 2 $2 b$ it is sufficient to choose $\delta$ small enough to achieve

$$
\frac{Q_{\beta}\left[\Psi_{\delta}\right]-E\left\|\Psi_{\delta}\right\|^{2}}{\left\|\Psi_{\delta}\right\|^{2}}<0
$$

and the claim of the theorem follows.

Validity of the above result can be extended also to the critical case when the integral in (15) vanishes, however, we need a somewhat stronger assumption on the regularity of $\dot{\theta}$. We also have to suppose that the twisting is "not fully reverted" by the perturbation. 
Theorem 3. Assume that $\omega$ is not rotationally symmetric and let $\dot{\theta}(\cdot)$ be given by (19). Suppose in addition that $\dot{\theta}(s)+\beta_{0}>0$ holds for $|s| \leq s_{0}$, and that $\ddot{\theta}$ exists and is of the class $L^{2}\left(\left[-s_{0}, s_{0}\right]\right)$. Let

$$
\int_{-s_{0}}^{s_{0}}\left(\dot{\theta}^{2}(s)-\beta_{0}^{2}\right) \mathrm{d} s=0
$$

then the operator $H_{\beta}$ has at least one eigenvalue of finite multiplicity below the threshold of the essential spectrum.

Proof. Following again the idea of 9] we improve the trial function used in the proof of Theorem 2 by a deformation in the central region,

$$
\Psi_{\delta, \gamma}(s, t):=f(t) \varphi_{\gamma}(s),
$$

where

$$
\varphi_{\gamma}(s)= \begin{cases}e^{\delta\left(s_{0}+s\right)} & \text { if } \quad s \leq-s_{0} \\ 1+\gamma\left(\beta_{0}-\dot{\theta}(s)\right) & \text { if } \quad-s_{0} \leq s \leq s_{0}, \\ e^{-\delta\left(s-s_{0}\right)} & \text { if } s \geq s_{0} .\end{cases}
$$

with $\gamma>0$. Similarly as in the proof of Theorem 2 one can check that

$$
Q_{\beta}\left[\Psi_{\delta, \gamma}\right]-E\left\|\Psi_{\delta, \gamma}\right\|^{2}=\int_{\mathbb{R} \times \omega}\left(\varphi_{\gamma}^{2}\left(f_{\tau}^{\prime}\right)^{2}\left(\dot{\theta}^{2}(s)-\beta_{0}^{2}\right)+f^{2}\left(\varphi_{\gamma}^{\prime}\right)^{2}\right) \mathrm{d} s \mathrm{~d} t .
$$

Using the assumptions of the theorem we find that the integrals appearing in the last expression behave as

$$
\int_{-s_{0}}^{s_{0}} \varphi_{\gamma}^{2}\left(\dot{\theta}^{2}(s)-\beta_{0}^{2}\right) \mathrm{d} s=-2 \gamma \int_{-s_{0}}^{s_{0}}\left(\dot{\theta}(s)-\beta_{0}\right)^{2}\left(\dot{\theta}(s)+\beta_{0}\right) \mathrm{d} s+\mathcal{O}\left(\gamma^{2}\right),
$$

and

$$
\int_{\mathbb{R}}\left(\varphi_{\gamma}^{\prime}\right)^{2} \mathrm{~d} s=\delta+\gamma^{2} \int_{-s_{0}}^{s_{0}}(\ddot{\theta}(s))^{2} \mathrm{~d} s=\mathcal{O}\left(\gamma^{2}\right)+\mathcal{O}(\delta) .
$$

as $\gamma, \delta \rightarrow 0$; the last two equations then give

$$
\begin{aligned}
\frac{Q_{\beta}\left[\Psi_{\delta, \gamma}\right]-E\left\|\Psi_{\delta, \gamma}\right\|^{2}}{\left\|\Psi_{\delta, \gamma}\right\|^{2}}= & -2 \gamma \delta \frac{\left\|f_{\tau}^{\prime}\right\|_{L^{2}(\omega)}^{2}}{\|f\|_{L^{2}(\omega)}^{2}} \int_{-s_{0}}^{s_{0}}\left(\dot{\theta}(s)-\beta_{0}\right)^{2}\left(\dot{\theta}(s)+\beta_{0}\right) \mathrm{d} s \\
& +\delta \mathcal{O}\left(\gamma^{2}\right)+\mathcal{O}\left(\delta^{2}\right) .
\end{aligned}
$$

Setting now $\gamma=\sqrt{\delta}$ we see that it is enough to take $\delta$ small enough to get

$$
\frac{Q_{\beta}\left[\Psi_{\delta, \gamma}\right]-E\left\|\Psi_{\delta, \gamma}\right\|^{2}}{\left\|\Psi_{\delta, \gamma}\right\|^{2}}<0
$$

which concludes the proof. 


\section{Acknowledgments}

The research has been partially supported by Czech Academy od Sciences and its Grant Agency within the projects IRP AV0Z10480505 and A100480501, and by DAAD within the project D-CZ 5/05-06.

\section{References}

[1] D. Borisov, P. Exner, R. Gadyl'shin, D. Krejčiř́ik, Bound states in weakly deformed strips and layers, Ann. H. Poincaré 2 (2001), 553-572.

[2] B. Chenaud, P. Duclos, P. Freitas, and D. Krejčiřík, Geometrically induced discrete spectrum in curved tubes, Differential Geom. Appl., to appear; math.SP/0412132.

[3] P. Duclos and P. Exner, Curvature-induced bound states in quantum waveguides in two and three dimensions, Rev. Math. Phys. 7 (1995), 73-102.

[4] T. Ekholm and H. Kovař́ík, Stability of the magnetic Schrödinger operator in a waveguide, Comm. PDE, to appear.

[5] T. Ekholm, H. Kovařŕk, and D. Krejčiřík, Twisting vs. bending in a quantum waveguide, in preparation.

[6] P. Exner, P. Freitas, and D. Krejčiřík, A lower bound to the spectral threshold in curved tubes, Roy. Soc. London Proc. Ser. A Math. Phys. Eng. Sci. 460 (2004), no. 2052, 3457-3467.

[7] P. Exner and P. Šeba, Bound states in curved quantum waveguides, J. Math. Phys. 30 (1989), 2574-2580.

[8] D. Gilbarg, N.S. Trudinger, Elliptic partial differential equations of second order, Springer-Verlag, Berlin 1998.

[9] J. Goldstone and R. L. Jaffe, Bound states in twisting tubes, Phys. Rev. B 45 (1992), 14100-14107.

[10] T. Kato, Perturbation theory for linear operators, Springer-Verlag, Berlin 1966.

[11] J. T. Londergan, J. P. Carini, and D. P. Murdock, Binding and scattering in two-dimensional systems, LNP, vol. m60, Springer, Berlin 1999.

[12] M. Reed and B. Simon, Methods of Modern Mathematical Physics IV. Analysis of operators, Academic Press, New York 1978. 
[13] W. Renger and W. Bulla, Existence of bound states in quantum waveguides under weak conditions, Lett. Math. Phys. 35 (1995), 1-12. 